\title{
Research on Economic Procurement Models on the Basis of Controllable Lead Time
}

\author{
Xiaoping Fang, Xianyun Li \& Li He \\ School of Traffic and Transportation Engineering, Central South University \\ Changsha 410075, China \\ E-mail: fangxp@csu.edu.cn
}

The study is sponsored by Hunan natural science fund, 09JJ3135. Role Principal Investigator

\begin{abstract}
On the basis of extremum principle, a mathematical model of two-echelon supply chain for both the suppliers and the purchasers is established. The model is built to analyze the decentralized and centralized procurement decision-making under the condition of controllable lead time and the joint undertaking of costs. By using the numerical analysis and operating the two models, the difference of the amount of economic procurement and the lead time under different manner of decision-making and costs undertaking are discussed. The results show that the amount of costs reduction is associated with the compensation costs paid by suppliers for further cutting down the lead time. Therefore, cutting down the lead time to a certain extent would reduce the overall costs of supply chain.
\end{abstract}

Keywords: SCM (Supply Chain Management), Lead time, Decentralized decision-making, Co-determination

\section{Preface}

The stock management, including ordering in advance and the confirmation of the amount of procurement within the supply chain is an important part of supply chain management (SCM). The costs of stocking and advance in time are closely related with each other. Lead time refers to the length of time from the issuing the order to the receiving the goods. It includes the time of four links: processing the order form, supply position, transportation and identifying the goods.

Many stock models regard the lead time as a constant or random variability (Luo, 2004) without considering the controllability of lead time and the influence it exerts on the amount of procurement and the safety of stock. In the production activity, the lead time could be cut down and controlled by supplementing the extra costs for operating around the clock.

Liao and Shyu (1991) are the first ones to research the controllable lead time. They assume that it is formed by many operation periods and every operation period contains an average lasting time and a minimal lasting time. Moreover, the extent of reduced lead time in every period changes with the costs in linear. Based on their research, Ben-daya \& Raouf's (1994) model regards both the lead time and the amount of ordering as decision-making variations. And Ouyang, Yen \& Wu (1996) put forward the model of lead time considering the condition of short supply and further categorize the shortage into two kinds: replenish-able short supply and the shortage that leads to loss.

However, thanks to the fact that the reduction of the costs of lead time is based on the assumption of linear change, there are still some deficiencies in practice. According to the principle that the marginal utility is actually a diminishing process, the costs of lead time in every operation period should exhibit a relationship of convex function. And after being minimized into linear, all the results of the mentioned dissertations are that the annual costs are at the lowest point when every single operation period of lead time is valued from the end points (it means the shortest duration and also the longest duration). The fact that the end point of optimal lead time only values the decentralized one which leaves us no chance to compare and analyze the function of reducing the lead time in stock management. Therefore, their results are not applicable in practice.

The thesis believes that the lead time can be cut down and controlled by supplementing costs. By applying the negative index function proposed in document 5 to deal with the compensation costs for cutting down the delivery lead time, 
which means that the time for cutting down the lead time varies with the costs in non-linear, the thesis builds two stock decision-making models including centralized and decentralized ones in the two-echelon supply chain.

\section{The Hypothesis of Models and Explanation of Signs}

\subsection{Basic Hypothesis}

A two-echelon supply chain between a purchaser and a supplier is taken into consideration. The purchaser sends the demand information through the order. The supplier should design supply strategies according to the demand information and their own conditions. The following hypothesis is made to resolve the problem:

(1) If the demand during the lead time follows the normal distribution of mean $\mu$ and Standard Deviation $\sigma$, the demand $X$ during a fixed lead time $l$ must follow the normal distribution of mean $\mu \cdot l$ and standard deviation $\mu \cdot l$.

(2) The reorder point $r=\mu \cdot l+k \sigma \sqrt{l}$, among which, $\mu \cdot l$ is the demand expectation during the lead time, $k \sigma \sqrt{l}$ the safety storage, $k$ the safe inventory factor, $k$ accords with $P(X>r)=P(Z>k)=q . Z$ is the random variable of the standard normal distribution and $k$ can be calculated in the normal distribution chart (Ling,2008).

(3) The inventory level is supervised in a sequential way so that when the storage lowers to the reorder point the immediate purchase form the supplier can be realized.

(4) The total annual demand is known and out of stock is unallowable.

\subsection{Explanation of Signs}

$D$ is total demand facing the purchaser in unit time; A stands for the purchaser's cost per order; Q stands for Buyer's order quantity, which is a decisive variable; $r$ is re-order point of the purchaser, which is a decisive variable; $h_{\mathrm{V}}$ shows the cost of purchaser's unit inventory holding per unit time; 1 is the normal procurement lead-time of the purchaser ; $\mathrm{L}$ stands for volume of compressed lead-time, which is a decisive variable; $\operatorname{CS}(L, Q)$ is the expected total cost per unit time of the purchaser; $\operatorname{Cv}(L, Q)$ shows the expected total cost per unit time of the supplier $\mathrm{C}(L, Q)$ stands for the joint expected total cost of both supplier and purchaser.

\section{Cost Analysis when lead time being a constant}

In the purchasing model the lead time is usually decided by the average replenishment time, that is to say the lead time is a constant in the model and does not affect the decisive variable. The purchaser determines the quantity of economic purchasing according to their total demand and safety stock. Thus the total cost is equal to:

$$
\operatorname{CS}(Q)=A \frac{D}{Q}+h\left(\frac{Q}{2}+k \sigma \sqrt{l}\right)
$$

Then $Q^{*}=\sqrt{\frac{2 A D}{h}}$, therefore $\operatorname{Cs~}\left(Q^{*}\right)=\frac{\sqrt{2 A D h}}{2}+h\left(\frac{1}{2} \sqrt{\frac{2 A D}{h}}+k \sigma \sqrt{l}\right)$

The inventories of the supplier equal to the purchasing quantity of the purchaser for a two-echelon supply chain. The cost of the supplier consists of inventory cost and preparation cost:

$$
\begin{aligned}
& \operatorname{Cv}(Q)=D S_{v} / Q+Q h_{v} / 2 \\
& \text { Then } Q^{*}=\sqrt{\frac{2 A D}{h}} \text {,i.e., } \operatorname{C~v}\left(Q^{*}\right)=\frac{S_{v} \sqrt{2 D A h}}{2 A}+\sqrt{\frac{2 A D}{h} h_{v}}
\end{aligned}
$$

\section{Cost Analysis of Controllable Procurement Lead Time}

Lead time control, and procurement lead time control in particular, plays a vital role that can not be neglected in the inventory management in enterprises. Shortening the lead time can reduce the amount of safety stock and inventory capital, thus to enhance the competitiveness of enterprises. However, this will increase ordering frequency, and thus increase set-up costs. So it should be considered carefully. In this thesis, the additional cost required by the shortened delivery lead-time adopts the form of exponential function: $C(L)=a e^{-b L}$. The cost compressed lead-time should be shared by the purchaser and supplier. There are two decision-making modes for the quantity of economic purchasing and lead time. The first one is de co-determination, and the second one is co-determination. They have different decision-making process.

\subsection{Co-determination Process and Cost Analysis}

The purchaser is in charge of determining the purchasing quantity in the co-determination process and the purchasing quantity is determined by the supplier in the lead time.

(1) The Determination of Economic Purchasing Quantity

The costs of purchaser are composed of purchasing cost, inventory cost and the cost formed in sharing the compressed 
lead time.

$$
C S(L, Q)=A \frac{D}{Q}+h\left(\frac{Q}{2}+k \sigma \sqrt{l+L}\right)+\frac{D}{Q} \alpha C(L)=A \frac{D}{Q}+h\left(\frac{Q}{2}+k \sigma \sqrt{l+L}\right)+\frac{D}{Q} \alpha a e^{-b L}
$$

$\alpha$ stands for the cost proportion of the purchaser. So the purchaser decides the quantity of economic purchasing according to a reasonable lead time provided by the supplier to minimize the total cost $\operatorname{CS}(L, Q)$. Therefore derivation of $Q^{*}$ can be obtained in the solution of the model. $Q^{*}=\sqrt{2 \frac{A D+D \alpha a e^{-b L}}{h}}$, It can be seen from that economic purchasing quantity of the purchaser and purchasing lead time are relevant to the sharing proportion of the cost of compressed lead time.

$$
\operatorname{CS}\left(L, Q^{*}\right)=A \frac{\sqrt{2 D h\left(A+\alpha a e^{-b L}\right)}}{2\left(A+\alpha a e^{-b L}\right)}+h\left(\sqrt{\frac{A D+D \alpha a e^{-b L}}{2 h}}+k \sigma \sqrt{l+L}\right)+\frac{\sqrt{2 D h\left(A+\alpha a e^{-b L}\right)}}{2\left(A+\alpha a e^{-b L}\right)} \alpha a e^{-b L}
$$

(2) Determination of Lead Time

Cost of the supplier consists of order cost, inventory cost and lead time shortening, so:

$$
\operatorname{Cv}(L, Q)=D S_{v} / Q+Q h_{v} / 2+\beta D C(L) / Q=S_{v} D / Q+Q h_{v} / 2+\beta a e^{-b L} D / Q
$$

Supplier's stock is determined by purchaser's need, that is to say purchaser places an order and the supplier prepare goods according to the order to reduce the cost.

For

$$
\begin{aligned}
Q^{*} & =\sqrt{2 \frac{A D+D \alpha a e^{-b L}}{h}} \\
\operatorname{So,} \mathrm{Cv}_{\left(L, Q^{*}\right)} & S_{v} D / \sqrt{2 \frac{A D+D \alpha a e^{-b L}}{h}}+\sqrt{2 \frac{A D+D \alpha a e^{-b L}}{h}} h_{v} / 2+\beta a e^{-b L} D / \sqrt{2 \frac{A D+D \alpha a e^{-b L}}{h}} \\
& =\left(S_{v}+\beta a e^{-b L}\right) \sqrt{D h} / \sqrt{2\left(A+\alpha a e^{-b L}\right)}+\sqrt{2 \frac{A D+D \alpha a e^{-b L}}{h}} h_{v} / 2 \\
\frac{\partial \mathrm{C}_{v}\left(L, Q^{*}\right)}{\partial L} & =\frac{\sqrt{2}}{4} S_{v} D^{\frac{1}{2}} \alpha b a e^{-b L} h^{\frac{1}{2}}\left(A+\alpha a e^{-b L}\right)^{-\frac{3}{2}}-\frac{\sqrt{2}}{4} \mathrm{D}^{\frac{1}{2}} h_{v} \alpha b a e^{-b L} h^{-\frac{1}{2}}\left(A+\alpha a e^{-b L}\right)^{-\frac{1}{2}} \\
& +\frac{\sqrt{2}}{4} D^{\frac{1}{2}} b \alpha \beta a^{2} e^{-2 b L} h^{\frac{1}{2}}\left(A+\alpha a e^{-b L}\right)^{-\frac{3}{2}}-\frac{\sqrt{2}}{2} D^{\frac{1}{2}} b \beta a e^{-b L}\left(\frac{A+\alpha a e^{-b L}}{h}\right)^{-\frac{1}{2}}
\end{aligned}
$$

Supposing (5) equals zero, so we can get

$$
L^{*}=-\log \left[\frac{S_{v} h \alpha-2 A h_{v} \alpha-2 A h \beta}{\left(h_{v} \alpha+\beta h\right) \alpha a}\right] / b
$$

When $\mathrm{L}<\mathrm{L}_{1}^{*}$, then $\frac{\partial \mathrm{C}_{\mathrm{v}}\left(L, Q^{*}\right)}{\partial L}<0$; when $\mathrm{L}>\mathrm{L}_{1}^{*}$, then $\frac{\partial \mathrm{C}_{\mathrm{v}}\left(L, Q^{*}\right)}{\partial L}>0$.

When $\mathrm{L}=\mathrm{L}_{1}^{*}, \operatorname{Cv}\left(L_{1}^{*}, Q^{*}\right)$ is the smallest.; When $\mathrm{L}^{*}<0$, let $\mathrm{L}^{*}=0$, that is to say the lead time is not shortened.

So $Q^{*}=\sqrt{2 \frac{A D+D\left(\frac{S_{v} h \alpha-2 A h_{v} \alpha-2 A h \beta}{\left(h_{v} \alpha+\beta h\right)}\right)}{h}}, L^{*}=-\log \left[\frac{S_{v} h \alpha-2 A h_{v} \alpha-2 A h \beta}{\left(h_{v} \alpha+\beta h\right) \alpha a}\right] / b$

When we put $Q^{*}$ 和 $L^{*}$ into $\operatorname{CS}(L, Q)$ and $\operatorname{Cv}(L, Q)$ respectively, we can get the lowest cost of purchaser and 
supplier.

$$
\begin{gathered}
\operatorname{CS}\left(L_{1}^{*}, Q^{*}\right)=\left(A+\alpha a e^{-b L}\right) \sqrt{2 D h} / 2 \sqrt{A+\alpha a e^{-b L}}+\sqrt{\left(A D+D \alpha a e^{-b L}\right) h / 2}+h k \sigma \sqrt{L} \\
=\left(A+\frac{S_{v} h \alpha-2 A h_{v} \alpha-2 A h \beta}{\left(h_{v} \alpha+\beta h\right)}\right) \sqrt{2 D h} / 2 \sqrt{A+\frac{S_{v} h \alpha-2 A h_{v} \alpha-2 A h \beta}{\left(h_{v} \alpha+\beta h\right)}} \\
+\sqrt{\left(A D+D \frac{S_{v} h \alpha-2 A h_{v} \alpha-2 A h \beta}{\left(h_{v} \alpha+\beta h\right)}\right) h / 2}+h k \sigma \sqrt{-\log \left[\frac{S_{v} h \alpha-2 A h_{v} \alpha-2 A h \beta}{\left(h_{v} \alpha+\beta h\right) \alpha a}\right] / b} \\
\operatorname{Cv}\left(L_{1}^{*}, Q^{*}\right)=\left[S_{v}+\beta \frac{S_{v} h \alpha-2 A h_{v} \alpha-2 A h \beta}{\left(h_{v} \alpha+\beta h\right) \alpha}\right] \sqrt{D h} / \sqrt{2\left[A+\left(\frac{S_{v} h \alpha-2 A h_{v} \alpha-2 A h \beta}{\left(h_{v} \alpha+\beta h\right)}\right)\right.} \\
+\sqrt{\frac{A D+D\left(\frac{S_{v} h \alpha-2 A h_{v} \alpha-2 A h \beta}{\left(h_{v} \alpha+\beta h\right)}\right)}{h}} h_{v} / 2
\end{gathered}
$$

\subsection{Co-determination Process and Cost Analysis}

If there is need for codetermination, supplier and purchaser should share their information to avoid such situation as inaccurate decision because of information asymmetry, lead time for purchase prolonged and high cost. The cost of the entire chain is much smaller than the sum of the cost of the purchaser and that of the supplier. Then the total cost is:

$$
\begin{aligned}
\mathrm{C}(L, Q)=D S_{v} / Q+ & +Q h_{v} / 2+\beta D C(L) / Q+A \frac{D}{Q}+h\left(\frac{Q}{2}+k \sigma \sqrt{l+L}\right)+\frac{D}{Q} \alpha C(L) \\
& =\frac{Q h_{v}}{2}+\left(S_{v}+A\right) \frac{D}{Q}+h\left(\frac{Q}{2}+k \sigma \sqrt{l+L}\right)+\frac{D}{Q} a e^{-b L}
\end{aligned}
$$

Different from getting lowest cost of purchaser and supplier through decentralized decision making, centralized decision making has an optimized lead time and economic purchasing amount, so both $\mathrm{L}$ and $\mathrm{Q}$ are decisive variable.

According to (8) we can get:

$$
\begin{gathered}
\left\{\begin{array}{l}
\frac{\partial \mathrm{C}(L, Q)}{\partial \mathrm{Q}}=\frac{h_{v}}{2}-\frac{\left(S_{v}+A\right) \mathrm{D}}{Q^{2}}+\frac{h}{2}-\frac{D a e^{-b L}}{Q^{2}}=0 \\
\frac{\partial \mathrm{C}(L, Q)}{\partial \mathrm{L}}=\frac{1}{2} h k \sigma(l+L)^{-\frac{1}{2}}-\frac{D}{Q} a b e^{-b L}=0
\end{array}\right. \\
\text { And }\left\{\begin{array}{l}
Q=\frac{-4 h_{v} D a^{2} e^{-2 b L}(l+L)+A h^{2} k^{2} \sigma^{2} a^{2} b^{2} e^{-2 b L} h l-2 D a^{2} b^{2} e^{-2 b L} h l+a e^{-b L} h^{2} k^{2} \sigma^{2}}{\left(h^{2} k^{2} \sigma^{2}\right)} \\
\frac{2 D a b e^{-b L}(l+L)^{\frac{1}{2}}}{h k \sigma}-L=0
\end{array}\right.
\end{gathered}
$$

The numerical value can be got when you insert this formula in order window "matlab".

\section{Model Analysis}

With the establishment of the cost models of the purchaser, supplier and supply chain in both decentralized and centralized decision making, this thesis then makes a case study on the models. If it is assumed that there is a purchaser $S$ whose average demand $\mathrm{D}=1000 \mathrm{t} / \mathrm{y}$, cost-per-order A $=100 \mathrm{CNY} /$ times, $\mathrm{h}=30 \mathrm{CNY} /$ ton, $\mathrm{S}_{v}=600 \mathrm{CNY} /$ times, $\mathrm{h}_{v}=20 \mathrm{CNY} /$ ton, $\sigma=20, \mathrm{k}=2$, normal average lead time $\mathrm{L}=16$ days, $\mathrm{a}=20, \mathrm{~b}=0.02$.

Insert Table 1 here

The economic purchasing quantity, the cost of the purchase, the cost of supplier and the total cost of supply chain can be 
calculated in the above model when the lead time remains a constant. They are $81.649 t, 7249.5 \mathrm{CNY}, 8165.0 \mathrm{CNY}$ $5414.5 \mathrm{CNY}$ respectively. The economic purchasing quantity, lead time in the process of co-determination and the cost are shown in the table1. During the co-determination process the economic purchasing quantity is $109.0238 \mathrm{t}$ and the shortened period in the lead time is 2.042 days. The cost of purchaser and supplier can be seen in the table2. It is can be seen from table 1 and table 2 that shortening the led time can reduce the cost of entire supply chain in different degree and shortening the led time in co-determination can minimize the cost of entire supply chain. The conclusion made from table 1 is that the cost of the purchaser varies inversely with the degree of compression of lead time. When the purchasing quantity increases to such degree that the cost of compression will be greater than the normal cost, the purchaser will chose not to shorten the lead time. So the distribution of cost proportion of compressing lead time and the reduced total cost must be reasonable.

Insert Table 2here

\section{Conclusions}

This thesis studies issues like delivery lead time and the decision making about order quantity in the two-echelon supply chain composed of a purchaser and a supplier. Cost compression in the lead time adopts the non-linear model. Both the supplier and purchaser should bear the additional cost caused by shortening the lead time according to their own conditions in order to minimize the cost of the entire supply chain. This thesis also analyzes the minimum cost when lead time being a constant and a variable respectively. Discussing the process co-determination and that of centralized one as well as their influence on the cost when lead time being a variable, this thesis testifies the practicality and efficiency of the model through the numerical examples. The result demonstrates that compressing the lead time under co-determination is most conducive to minimization of the cost of the supply chain.

\section{References}

Ben-Daya M, \& Raouf A. (1994). Inventory Models Involving Lead Time as Decision Variable $\mathrm{C}(L, Q)$. Journal of the Operational Research Society.

BEN-DAYAM, RAOUFA. (1994). Inventory Models Involving Lead Time as Decision Variable. JOperationalRes Soc.

Ling, Yong. (2008). Inventory Management of Component Commonality in Supply Chain. Huazhong University of Science and Technology Press, in Chinese.

Liao C J, \& Shyu C H. (1991). An Analytical Determination of Lead Time with Normal Demand. International Journal of Operations and Production Management.

Luo, Bing. (2004). Study on Inventory Management of Supplier in Environment of Supply Chain. Industrial Engineering and Management, in Chinese.

Ouyang L Y, Yen N C, \& Wu K S. (1996). Mixture Inventory Model with Backorders and Lost Sales for Variable Lead Time. Journal of the Operational Research Society. 
Table 1. Economic Purchasing Quantity, Lead Time and Cost in co-determination Process

\begin{tabular}{|c|c|c|c|c|c|c|}
\hline$\alpha$ & $\beta$ & $\mathrm{Q}^{*}$ & $\mathrm{~L}^{*}$ & $\operatorname{Cs}(L, Q)$ & $\mathrm{Cv}(L, Q)$ & $\mathrm{C}(L, Q)$ \\
\hline 0.1 & 0.9 & 81.6497 & 0 & 7249.5 & 8165.0 & 15414.5 \\
\hline 0.2 & 0.8 & 81.6497 & 0 & 7249.5 & 8165.0 & 15414.5 \\
\hline 0.3 & 0.7 & 82.8846 & -1.9744 & 7019.2 & 8243.5 & 15262.7 \\
\hline 0.4 & 0.6 & 83.3289 & -1.9080 & 7054.5 & 8183.3 & 15237.8 \\
\hline 0.5 & 0.5 & 83.7435 & -1.7034 & 7111.1 & 8125.7 & 15236.8 \\
\hline 0.6 & 0.4 & 84.1380 & -1.5420 & 7160.5 & 8070.6 & 15231.1 \\
\hline 0.7 & 0.3 & 84.5386 & -1.4206 & 7203.3 & 8015.8 & 15219.1 \\
\hline 0.8 & 0.2 & 84.9375 & -1.3294 & 7241.1 & 7961.8 & 15202.9 \\
\hline 0.9 & 0.1 & 85.3346 & -1.2609 & 7275.2 & 7908.5 & 15183.7 \\
\hline
\end{tabular}

Table 2. Economic Purchasing Quantity, Lead Time and Cost in co-determination Process

\begin{tabular}{|c|c|c|c|c|c|c|}
\hline$\alpha$ & $\beta$ & $\mathrm{Q}^{*}$ & $\mathrm{~L}^{*}$ & $\mathrm{Cs}(L, Q)$ & $\mathrm{Cv}(L, Q)$ & $\mathrm{C}(L, Q)$ \\
\hline 0.1 & 0.9 & 109.0238 & -2.0420 & 7054.9 & 6765.6 & 13820.5 \\
\hline 0.2 & 0.8 & 135.0603 & -2.0420 & 7074.1 & 6746.5 & 13820.6 \\
\hline 0.3 & 0.7 & 135.0603 & -2.0420 & 7093.2 & 6727.4 & 13820.6 \\
\hline 0.4 & 0.6 & 135.0603 & -2.0420 & 7112.3 & 6708.3 & 13820.6 \\
\hline 0.5 & 0.5 & 135.0603 & -2.0420 & 7131.4 & 6689.2 & 13820.6 \\
\hline 0.6 & 0.4 & 135.0603 & -2.0420 & 7150.5 & 6670.1 & 13820.6 \\
\hline 0.7 & 0.3 & 135.0603 & -2.0420 & 7169.6 & 6651.0 & 13820.6 \\
\hline 0.8 & 0.2 & 135.0603 & -2.0420 & 7188.7 & 6631.8 & 13820.5 \\
\hline 0.9 & 0.1 & 135.0603 & -2.0420 & 7207.8 & 6612.7 & 13820.5 \\
\hline
\end{tabular}

